Review

\title{
Changes of Migration, Immunoregulation and Osteogenic Differentiation of Mesenchymal Stem Cells in Different Stages of Inflammation
}

\author{
Feng Gu, Ke Zhang, Jiangbi Li, Xiaoping Xie, Qiangqiang Wen, Zhenjiang Sui, Zilong Su, Tiecheng Yu ${ }^{\bowtie}$ \\ Department of Orthopedics, First Hospital of Jilin University, Changchun 130021, Jilin, China. \\ $\triangle$ Corresponding author: Tiecheng Yu, E-mail: yutc@jlu.edu.cn; Tel.: 0086-0431-88785691; Postal Address: 130021. \\ (c) The author(s). This is an open access article distributed under the terms of the Creative Commons Attribution License (https://creativecommons.org/licenses/by/4.0/). \\ See http://ivyspring.com/terms for full terms and conditions.
}

Received: 2021.01.20; Accepted: 2021.10.25; Published: 2022.01.01

\begin{abstract}
Bone infection has always been the focus of orthopedic research. Mesenchymal stem cells (MSCs) are the natural progenitors of osteoblasts, and the process of osteogenesis is triggered in response to different signals from the extracellular matrix. MSCs exert important functions including secretion and immune regulation and also play a key role in bone regeneration. The biological behavior of MSCs in acute and chronic inflammation, especially the transformation between acute inflammation and chronic inflammation, has aroused great interest among researchers. This paper reviews the recent literature and summarizes the behavior and biological characteristics of MSCs in acute and chronic inflammation to stimulate further research on MSCs and treatment of bone diseases.
\end{abstract}

Key words: Mesenchymal Stem Cells, Infection, Immunomodulatory, Differentiation

\section{Introduction}

Bone infection has always been the focus of orthopedic research. Bone infection is a serious complication in orthopedics and often leads to severe bone destruction and nonunion. When acute infection is not effectively controlled, it may become chronic, which often leads to disastrous consequences. Osteomyelitis is a typical bone infection, and it often requires prolonged antibiotic treatment and surgical intervention. Osteomyelitis is caused by bacterial pathogens, and Staphylococcus aureus and Staphylococcus epidermidis are responsible in most cases [1]. Mesenchymal stem cells (MSCs) originate from the mesoderm and are characterized by the potential of self-proliferation and multi-directional differentiation, and can differentiate into osteoblasts, chondrocytes, and adipocytes in vitro [2-4]. The potential of MSCs to differentiate into different tissue types has made the study of MSCs a hot research topic in recent years. MSCs are the natural progenitors of osteoblasts and are involved in the process of osteogenesis in response to different signals from the extracellular matrix (ECM), and play a critical role in the bone regeneration and immunity [5-7]. As a result, the study of the response of MSCs to inflammation and its different stages are of great interest.

During the process of bone regeneration, inflammatory factors can activate and mobilize endogenous MSCs, which migrate from their niche to the damaged site [8]. MSCs can promote the regeneration of bone tissue through osteogenic differentiation and are the key cells involved in fracture healing. However, infection often leads to progressive bone destruction and loss, and eventually bone nonunion occurs when inflammation persists and becomes chronic. Bone defects and nonunion caused by infection have always been difficult to treat clinically, not only because inflammation is difficult to control, but also because the differentiation and osteogenic ability of MSCs and osteoblasts is altered under a chronic state of inflammation. Acute and chronic inflammation are often associated with pathogens, and the interplay between infection and inflammation is of paramount importance to clinical outcomes [9]. Therefore, the migration, differentia- 
tion, immune regulation, secretion, and other functions of MSCs in acute and chronic inflammation have become an area worthy of study. Following a review of the literature, we summarized the biological performance of MSCs in acute and chronic inflammation. In infection and inflammation MSCs interact with immune cells to intricately regulate cellular activity. Differences in the behaviors of MSCs in acute and chronic inflammations have also become the focus of significant research attention. It is known that the transformation from acute inflammation to chronic inflammation is a key feature of tissue repair. The behaviors of MSCs in the acute and the chronic inflammations also suggest that if the mechanisms involved in the transformation between the two stages are better understood, intervention on MSCs activities in the infection would be made possible, and the basis for treating the bone infection be provided.

\section{Inclusion of references}

A literature search was conducted with PubMed, EMBASE and Web of Science databases. The following terms: ('mesenchymal stem cells', 'acute inflammation', 'chronic inflammation', 'migration', immunoregulation' and 'osteogenic'), were used to search eligible studies from 2002 to 2020, with 80 relevant English articles included in this review.

\section{Signal pathway related to osteogenesis of MSCs}

It is know from previous studies that in the process of regulating the osteogenic differentiation of MSCs, signaling pathways such as mitogen-activated protein kinases (MAPK) [10], bone morphogenetic protein (BMP)/mothers against decapentaplegic homolog (SMAD) [11-13], Wnt/ $\beta$-catenin $[14,15]$ and PI3K-Akt-Mtor [16] play a key role.

MAPK is a conserved family of enzymes that can transmit and spread external stimuli, and coordinate cell responses through phosphorylation cascades to adapt to environmental changes [10,17]. It is a prolinedirected serine/threonine (Ser/Thr) specific protein kinase that regulates cell activities, such as gene expression, mitosis, differentiation, and cell survival/ apoptosis. So far, four different types of mammalian MAPKs have been identified as extracellular regulated protein kinases 1 and 2 (ERK1 and 2), c-Jun N-terminal kinases 1-3 (JNK 1-3), p38MAPK (p38a, p38 $\beta$, p38 $\gamma$ and p386) and ERK5 [18]. Activated ERK, JNK and p38MAPK have different signal pathways, but there is also cross-regulation between the signal pathways. p38MAPK contains a set of 4 proteins, i.e., p38a, p38 $\beta, p 38 \gamma$ and p386, which represent different branches of the phylogenetic tree and are each conserved in species. Among the p38MAPK family, p38a is the most widely expressed and studied; $p 38 \beta$ and $\delta$ are expressed in immune cells, and p38y is a highly restricted expression which has not been fully elucidated yet [18]. Phosphorylated p38MAPK can activate activator protein 1 (AP-1), transcription factor 1 (SP-1) and NF-kB and other signaling pathways, and promote the secretion of various cytokines and inflammatory mediators. In the so-called classical p38MAPK signaling pathway, phosphorylation of p38MAPK in the cytoplasm can activate several transcription factors, including $\mathrm{AP}-1$ and $\mathrm{SP}-1$, regulate their movement to the nucleus, and then transcribe several inflammatory mediators and cytokines [18]. The AP-1 components, mainly members of the Fos family such as c-Fos and Fos related antigen-1 (Fra-1), have important functions in both of these cell types [19].

In addition, the phosphorylated p38MAPK activates mitogen and stress-activated protein kinases 1 (MSK 1) and mitogen and stress-activated protein kinases 2 (MSK 2), which can phosphorylate the NF-kB complex transactivating Ser276 of the p65 subunit, thereby enhancing the NF-kB signaling [18]. The phosphorylated AP-1, SP-1 and NF-kB increase the secretion and synthesis of a variety of inflammatory cytokines, and the body's immune defense system is thus activated [18].

BMPs are members of the transforming growth factor $\beta$ (TGF- $\beta$ ) superfamily [20]. They are powerful osteogenic differentiation factors in the process of bone formation and play an important regulatory role therein. Intracellular BMP signal transduction is mainly mediated by the SMAD protein. The SMAD protein binds to transmembrane receptor-threonine kinase through BMP and gets activated [20]. The activated SMADs are then transported to the nucleus, where they regulate the expression of genes involved in osteogenesis. BMP2 can induce bone formation and differentiation in vivo and in vitro. It activates SMAD1/5/8 and several mitogen-activated protein kinases (MAPK), which ultimately leads to the increase in the runt-related transcription factor 2 (RUNX2) and the core binding factor A1 (CBFA1) [20].

The SMAD protein family can be divided into 3 categories, the receptor-activated SMAD (R-SMAD), the common pathway SMAD (Co-SMAD) and the inhibitory SMAD (I-SMAD) [13]. R-SMAD can be activated by type I receptors and form transient complexes of receptors. It is further divided into two categories, the AR-SMAD activated by activin TGF- $\beta$, including SMAD 2, SMAD 3; and BMP. Other activated BR-SMAD includes SMAD 1, SMAD 5, SMAD 8, and SMAD 9 [30]. Co-SMAD includes SMAD 4, which is a common mediator required in various signal transduction processes of the TGF- $\beta$ 
family [13]. I-SMAD includes SMAD 6 and SMAD 7, which can bind to activated type I receptors to inhibit or regulate the signal transduction of the TGF- $\beta$ family [21]. The SMAD protein family plays a key role in the process of transmitting TGF- $\beta$ signals from cell surface receptors to the nucleus, and different SMADs mediate the signal transduction of different TGF- $\beta$ family members [13]. The receptor complex formed by TGF- $\beta$ as a ligand activates SMAD to enter the nucleus and jointly activate or inhibit the transcription of target genes regulated by them [21]. PI3K-AktmTOR signaling pathway regulates many key functions of cells [22]. Intracellular signaling mediates phosphoinositide 3-kinase (PI3K), Akt (protein kinase $\mathrm{B} / \mathrm{PKB}$ ) and mammalian target of rapamycin (mTOR) to form a signal network [22]. The members of this signaling network control the expression of proteins that regulate apoptosis and cell cycle of progression/ proliferation. They are very important for cell transportation/migration, and are also important regulators of cell metabolism and differentiation.

The $\mathrm{Wnt} / \beta$-catenin signaling pathway regulates the osteogenic differentiation, bone formation and bone metabolism disorders of bone marrow mesenchymal stem cells $[15,23]$. The activation of this typical pathway depends on the binding of Wnt, frizzled receptors and LRP co-receptors to the cell membrane, which will inhibit the expression of glycogen synthase kinase-3b (GSK3b) and promote the $\beta$-catenin migration to the nucleus [23]. In the nucleus, the $\beta$-catenin enhances the transcription regulated by TCF4 (T-cell factor 4)/LEF1 (lymphoid enhancing factor-1), and the $\beta$-catenin-TCF4 complex activates the downstream targets gene transcription [24].

\section{MSCs in the stage of acute inflammation}

\section{Migration ability of MSCs in acute inflammation}

MSCs will recruit and exert biological effects under the action of cytokines and chemokines in acute inflammation, and this process also allows the organism to heal from infections and tissue damage. Infection or trauma to tissues is usually detected by receptors largely located on the surface of innate immune cells, which are called pattern recognition receptors (PRRs). These PRRs then identify the pathogen-related molecular patterns or damageassociated molecular patterns and trigger the inflammatory response. Acute inflammation peaks at 24 to $48 \mathrm{~h}$ after injury and is complete after about 7 days [25-27]. MSCs undergo a process of selective migration to the site of injury and continuously secrete various nutritional factors, a function called "homing" [28]. MSCs are then selectively recruited to the damaged or inflammatory tissues, based on the release of multiple inflammatory factors by the injured tissues [29]. This process is essential for healing of the infection and bone regeneration. The migration ability of MSCs is increased in the acute inflammation, a process related to the release of tumor necrosis factor- $\alpha$ (TNF- $\alpha$ ) and the activation of the NF-kB pathway $[30,31]$. Ward et al. suggested that the up-regulation of podoplanin in the infected state is associated with the increased migration ability of MSCs [32]. Toll-like receptors (TLRs) are a family of PRRs and are responsible for identifying the exogenous pathogens. TLRs are mainly expressed by first-line immune cells, such as neutrophils, macrophages, and dendritic cells (DCs). These receptors, which belong to the innate immune pathway, can recognize different microbial structures and induce various immune responses to specific pathogens. TLRs are expressed not only by immune cells, but also by MSCs. MSCs directly recognize pathogens through the TLRs receptors expressed on the cell surface and regulate the biological response of the cells to the acute inflammation. TLRs are activated by their respective endogenous ligands; for example, TLR-2 recognizes lipoteichoic acid (LTA), TLR-4 recognizes lipopolysaccharide (LPS), and TLR-5 recognizes flagellin, which leads to the activation of MSCs and to the generation and release of cytokines and chemokines $[28,33]$. The activation of TLRs may affect the migratory function of MSCs. The TLRs expressed by MSCs from different sources may differ [33], and the activation of TLRs may not always play a positive role in the migration of MSCs. Although, in most cases, TLRs will enhance the migration of MSCs, there are studies indicating that TLR-3 inhibit migration [34,35]. The change of migration status of MSCs in infection is complex, and the function of TLRs is worthy of further study to understand the underlying mechanisms.

\section{Osteogenesis differentiation of MSCs in acute inflammation}

In the presence of acute infection, the acute inflammation response plays an important role in bone regeneration, and it is a crucial first step for bone healing. By contrast, the absence and inhibition of an acute inflammatory response may lead to impaired bone healing [27]. The outcome of an appropriate acute inflammation response in the early stages is favorable to osteogenesis, although the response may also switch to a negative regulatory effect when "over-stimulated". Previous studies have shown that drugs such as non-steroidal anti-inflammatory drugs (NSAIDs), chemotherapeutic agents and cortico- 
steroids may increase the risk for nonunion [36,37]. However, an excessive acute inflammatory response will increase the risk for impaired bone regeneration [38]. The specific regulatory mechanisms involved in the acute inflammatory response on osteogenic differentiation warrants more in-depth study. If the extent to which an "appropriate" inflammatory response could be determined, then the mechanism of bone healing in the state of infection and trauma would be more clearly defined. The osteogenic differentiation of MSCs is associated with multiple pathways, and the mechanism involved is very complex (Fig. 1). When the bone is infected, the inflammatory response can regulate the osteogenic differentiation of MSCs in different ways. The main feature of acute infection is the release of proinflammatory immune factors. We found that the differentiation of MSCs in acute inflammation may proceed in different directions and induce both promotion and inhibition of bone formation. Under certain conditions, the inflammatory response will enhance the osteogenesis of MSCs. For example, studies have shown that low-dose Staphylococcus aureus exotoxin stimulation will enhance the osteogenesis of MSCs, and LTA stimulation will also promote the bone formation of MSCs [39,40]. However, when the inflammatory response continues to increase, the osteogenic differentiation of MSCs begins to weaken, and apoptosis is increased. We know that proinflammatory factors will activate the NF-KB signaling pathway in acute inflammation, and activation of this pathway will promote $\beta$-catenin degradation and inhibit osteogenic differentiation [41]. Several studies [42-44] have shown that the activation of the NF-kB pathway has an adverse effect on the osteogenic differentiation of MSCs [42-44]. Xu et al. showed that the purinergic receptor $\mathrm{p} 2 \mathrm{x}$ and ligand gated ion channel, 7 (P2X7) receptors are also key molecules that regulate the osteogenesis of MSCs in acute inflammatory conditions [45]. P2X7 is a receptor located on the surface of cell membranes, which can be activated by adenosine triphosphate (ATP) and then can activate downstream MAPK receptors [46]. The MAPK pathway is mainly divided into three cascades: the ERK 1/2, p38 MAPK, and c-Jun N-terminal kinase (JNK) pathways. Although there are differences in cellular function, all MAPKs signal through a similar mechanism of associated proteins and activate common downstream transcription factors, known as AP-1, RUNX2, and recombinant osterix (OSX) [47]. Previous research reported here suggest that $\mathrm{P} 2 \mathrm{X} 7$ receptor agonist directs differentiation toward the osteoblast lineage and away from the adipocyte lineage in MSCs by stimulating ERK1/2 and JNK signaling pathways in a P2X7R dependentway [46]. P2X7R contributes to homeostatic control of MAPK cascades and shape the outcome of ERK signaling. P2X7R participates in feedforward loops to prolong ERK activation towards its cytoplasmic substrates [48,49]. In the acute inflammatory state, expression of the $\mathrm{P} 2 \mathrm{X} 7$ receptor will be significantly reduced and thus will reduce the osteogenic differentiation of MSCs $[45,46]$.

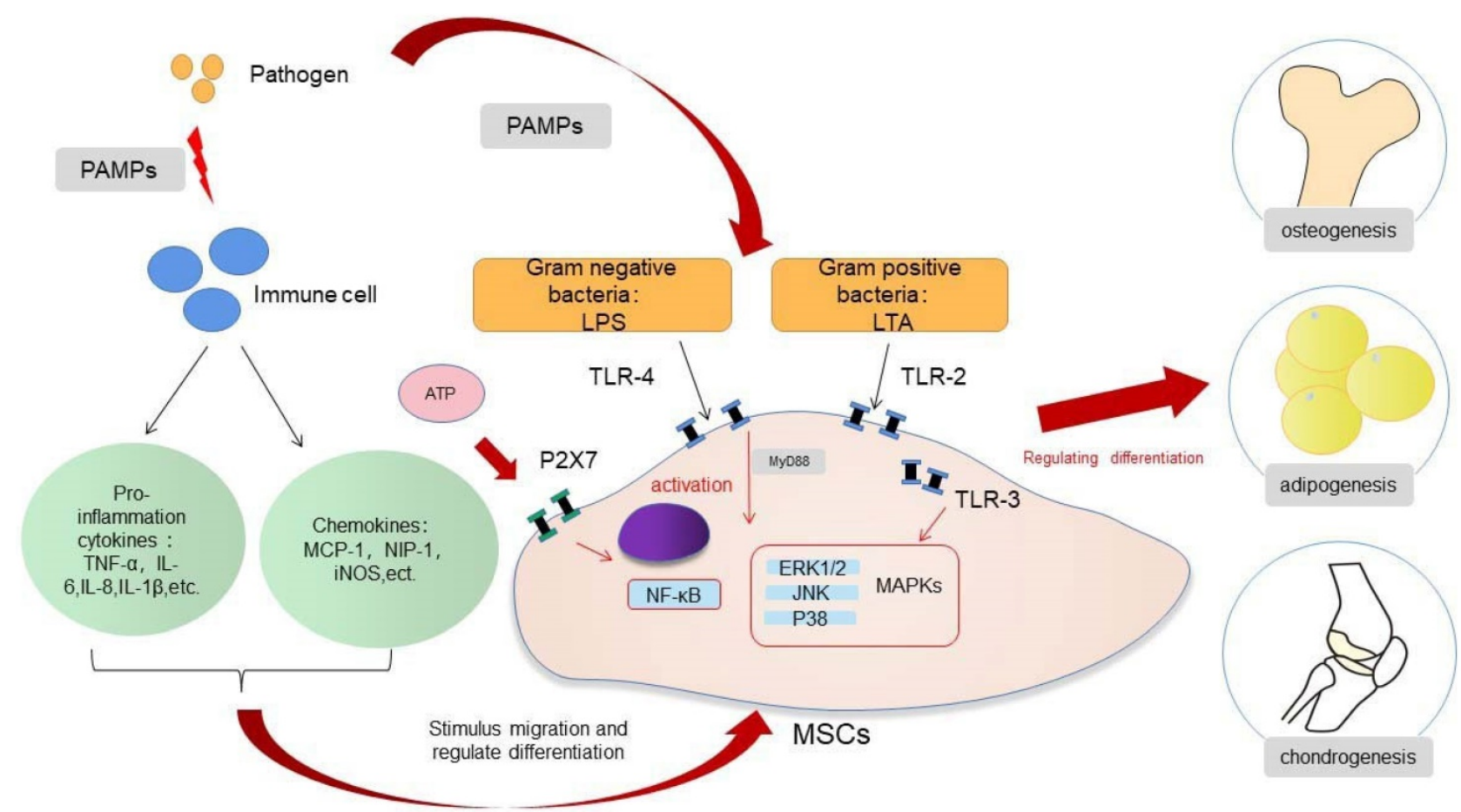

Figure 1. Regulation of MSCs differentiation in inflammation 


\section{Osteogenesis differentiation of MSCs in heterotopic ossification}

In the process of inflammatory response, the same cytokine will have different regulatory effects on the osteogenic differentiation of MSCs under different concentration and action time conditions. Studies have shown that low concentration of TNF- $\alpha$ (1 $\mathrm{ng} / \mathrm{ml}$ ) promotes the osteogenic differentiation of MSCs in the short term ( $1 \mathrm{~d}$ and $3 \mathrm{~d}$ ) and increases the mRNA expression of BMP-2 and SMAD 1, but inhibits the osteogenic differentiation of MSCs at long term (7 d). However, whether long-term or short-term, high concentrations of TNF-a inhibited the osteogenic differentiation of MSCs and the expression of SMAD 1 , but resulted in high expression of BMP-2 [50].

Very similarly, studies have shown that IL-10 has concentration-dependent, dual roles in the osteogenesis of MSCs through P38MAPK and NF-kB signaling pathways. Under low-dose conditions (0.1-1 $\mathrm{ng} / \mathrm{ml}$ ), it can promote osteogenesis. But when the concentration of IL-10 exceeds $10 \mathrm{ng} / \mathrm{ml}$, it will inhibit osteogenesis [51]. Such experimental results are also consistent with the different manifestations of MSC's osteogenic ability in both acute inflammation and chronic inflammation.

Among the ossification caused by inflammation, heterotopic ossification is a very special type of decease, which we will separately describe in detail. Heterotopic ossification (HO) often occurs after severe trauma or burn [52] and the process during which $\mathrm{HO}$ forms is governed by MSCs that are attempting to regenerate tissue based on cues from the inflammatory niche [53]. Heterotopic ossification often occurs primarily through endochondral ossification, which shows its common feature [53]. Evans et al included 24 cases of 36 high-energy penetrating injuries, of which 13 (36\%) had heterotopic ossification. After debridement, the tissue fragments and local exudate were tested for inflammatory factors and found that IL-6, IL-10 and monocyte chemoattractant protein-1 are significantly related to the occurrence of the heterotopic ossification [54]. Transforming growth factor- $\beta 1$ (TGF- $\beta 1$ ) is also known as a critical regulator in this process $[55,56]$. Formation of $\mathrm{HO}$ has been shown to require the recruitment of macrophages and mast cells for genetic and neurogenic HO [57-59]. Previous study revealed predetermination of aberrant chondrogenic cell fate of the mesenchymal progenitor cell population as early as 3 days after injury, indicating that the inflammatory phase is the critical time window for therapeutic targeting [53]. Although the formation process of heterotopic ossification is relatively slow, its mechanism does not seem to be the result of prolonged inflammatory response stimulation, but rather the gradual evolution of the inflammatory response after the acute trauma. MSCs were recruited to the injury site, and the environmental niche consisted of other cell types and microenvironmental factors are playing critical roles in influencing the fate of MSCs [60]. Therefore, the occurrence of the heterotopic ossification is related to the inflammatory response, but its outcome is not a simple regulation of the acute inflammation or chronic inflammation, rather, it is caused by more complex reasons. The specific mechanism still needs further research and discussion.

\section{Immunomodulatory properties of MSCs in acute inflammation}

In acute infections, MSCs mainly exert immunosuppressive effects and, under certain conditions, may also exhibit proinflammatory effects. MSCs induce a complex regulation of a variety of immune cells in acute infection and interact with immune cells through proinflammatory and immunosuppressive regulatory directions. MSCs have immunomodulatory functions, which are mainly achieved by interacting with different immune cell subpopulations [61]. T cells, B cells, natural killer (NK) cells, macrophages, dendritic cells (DCs) and other immune cells play an important role in our body, and MSCs can regulate their function (Fig. 2). MSCs mainly exert an immunosuppressive role in acute inflammation. T cells can be divided into helper $\mathrm{T}$ (Th) cells, cytotoxic $\mathrm{T}$ lymphocyte (CTL), and regulatory $\mathrm{T}$ cells (Tregs), according to their functions. Th cells can be divided into Th1, Th2, and Th17. Th1 can produce interleukin-2 (IL-2), interferon- $\gamma$ (IFN- $\gamma$ ), transforming growth factor- $\beta$ (TNF- $\beta$ ), and other immune factors, thereby activating macrophages and enhancing cellular immunity. Th2 can produce IL-4, IL-6, IL-10, and other products and activates B cells to enhance the humoral immunity. Tregs are a type of $\mathrm{T}$ cell with immunosuppressive effects [62]. The main features of the $\mathrm{T}$ cell response involve cell proliferation and cytokine secretion. Inhibition of the proliferation is the most significant effect of MSCs on T cells. In vitro, MSCs can suppress the proliferation of $\mathrm{T}$ cells induced by mitogens and alloantigens. Although the exact mechanisms underlying the immunosuppressive effects of MSCs are still unclear, most evidence indicates that soluble factors are involved. These factors include prostaglandin E2 (PGE2), indoleamine 2,3-dioxygenase (IDO), hepatocyte growth factor (HGF), IFN- $\gamma$, and TGF- $\beta 1$. MSCs can also alter the balance of Th1/Th2 cells [63] and $\mathrm{T}$ cell differentiation into Treg cells and downstream effects on acute inflammation and 
immune activity [64]. MSCs can inhibit the differentiation, proliferation, activation, and chemotaxis of B cells under the action of the C-C motif ligand 2 (CCL2) and PGE2, but under the influence of vascular endothelial growth factor (VEGF), they will promote the differentiation, proliferation, activation, and chemotaxis of B cells [65]. In acute inflammation, MSCs can mediate the transformation of M1 macrophages to the M2 type [66]. Extracellular vesicles of MSCs also regulate the activation of B cells [67]. In this process, the proinflammatory response mediated by TLR- 4 and the immunosuppressive effect mediated by TLR-3 produce a bidirectional regulation of $B$ cells and $T$ cells [28]. In acute inflammation, MSCs can mediate the transformation of M1 macrophages to the M2 type [68]. M1 macrophages exert a proinflammatory effect and can secrete various cytokines and chemokines, whereas M2 macrophages are anti-inflammatory cells that induce immune tolerance and promote cell repair and regulation. A recent study has shown that murine MSCs shift macrophages to an M2 phenotype through TGF- $\beta 1$ [69]. MSCs exerted this effect by inhibiting NF-kB p65 and by activating the signal transducers and activators of the transcription 3 (STAT3) pathways [70]. Interfering with the conversion of M1 to M2 macrophages may become a breakthrough for the treatment of infectious diseases in the future. The effects of MSCs are not always immunosuppressive; however, MSCs can adopt a proinflammatory state under certain conditions. For example, the presence of low levels of IFN- $\gamma$ and TNF can endow MSCs with immunostimulatory potential [71]. MSCs produce PGE2 under inflammatory conditions, and factors such as IL-10 can inhibit the maturation of DCs and produce an immunosuppressive effect [64]. Macrophage colony stimulating factor (M-CSF) inhibits the activation, differentiation, migration, endocytosis, and maturation of DCs during inflammation [72]. Only a few studies have investigated the interaction of NK cells with MSCs. In their pioneering work, Moretta et al. showed that resting unstimulated MSCs are capable of inhibiting NK cell proliferation, cytokine release, and cytotoxicity, via PGE2 and IDO [73]. More recently, studies have shown that although MSCs activated in an inflammatory state have a limited effect on NK cells, they can promote their differentiation [74].

\section{MSCs involvement in the stage of chronic inflammation}

In chronic inflammation, the immune regulatory function and osteogenic differentiation ability of MSCs are altered, which eventually leads to the persistence of inflammation, bone destruction, and nonunion. The inflammatory response is an essential and critical step in the healing process of acute bone infections; however, when the pathogen is difficult to remove, it will turn into chronic infection. Munir et al. believe that in chronic inflammation, MSCs residing

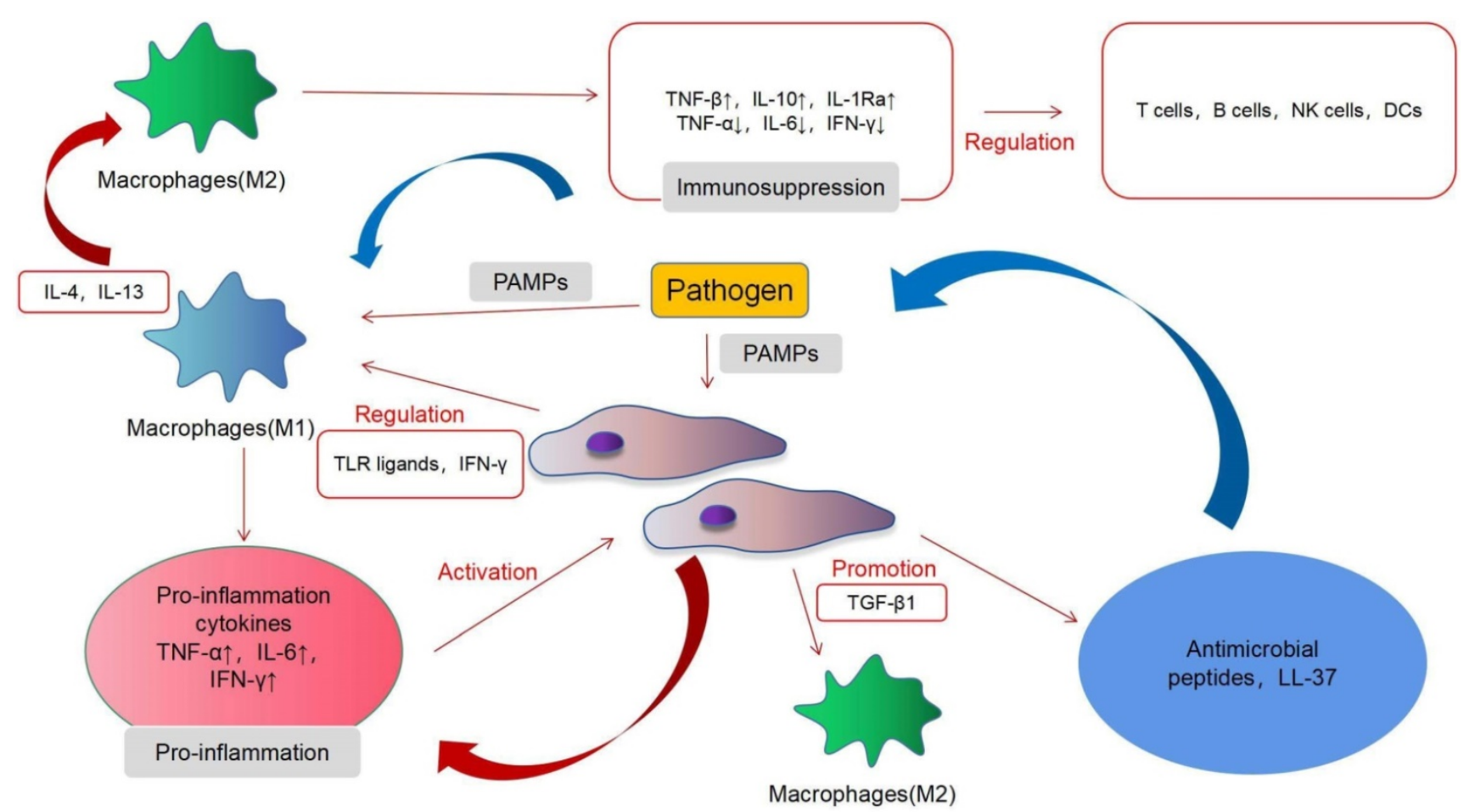

Figure 2. Immunomodulatory properties of MSCs in inflammation. 
in tissues will acquire a proinflammatory phenotype opposite to that of normal MSCs and will then produce proinflammatory factors [75]. This response can, to some extent, explain the nonunion and osteolysis observed in chronic osteomyelitis. In this process, bone tissue regeneration is accompanied by the repair of surrounding soft tissue damage. But in many cases, when the infection cannot be effectively controlled, the persistent inflammatory reaction will change from acute to chronic. In chronic inflammation, TNF- $\mathrm{a}$ and the NF-kB signaling pathways are continuously activated, which will induce the differentiation and activation of osteoclasts [27]. An acute inflammation that persists may shift into a chronic inflammatory state and persevere for a prolonged period. The continuous expression of the NF-kB pathway can reduce the osteogenesis and migration of MSCs and the apoptosis of MSCs [34,42]. M1 macrophage activation continuously produces cytokines, resulting in an imbalance of M1/M2 macrophages in chronic inflammation [76]. Bacteria adapt to and persist within chronic wounds as a biofilm, a sign of chronic bacterial infection. The presence of a biofilm will continue to release soluble factors. These soluble factors will induce further proliferation and differentially affect the differentiation of MSCs, including osteogenesis and adipogenesis, and induce the apoptosis of MSCs [34].

\section{MSC as a novel approach for infection treatment}

Because of the effective differentiation potential of MSCs and their immunoregulatory properties during the inflammatory response, MSCs have become highly regarded in tissue engineering, wound repair, immunotherapy, and infection treatment. Many studies have proposed MSCs as a treatment for infection. Saeedi et al. used MSCs primed by modified LPS to treat sepsis, and, in this context, MSCs presented strong antibacterial properties [77]. Yuan et al. used MSCs to reduce methicillin-resistant Staphylococcus aureus infection in rat models [78]. It is related to its immunomodulatory ability and to the production of antibacterial peptides LL-37 after stimulating MSCs [79]. The immune regulatory ability of MSCs makes it possible to regulate various immune cells in the body under the infection state and enhance their antibacterial ability. Alternatively, the production of antibacterial peptides is also an important factor for the antibacterial ability of MSCs. Under the activation of TLR-2 and TLR-4 receptors, MSCs can produce antibacterial peptides, which can kill pathogens directly. Simultaneously, MSCs can also affect immune regulation. Through the interaction with immune cells in the body, the bactericidal effects are enhanced to achieve the purpose of treating infection. However, MSCs are immature to treat infections, and a prior study has shown that when MSCs are implanted into non-sterile bone defects, their overall trophic activity may enhance an infection and/or exacerbate osteomyelitis, which might also be associated with its immunosuppressive effects [80]. Although the specific mechanisms are still not completely understood, the above-mentioned undesired effects undoubtedly suggest that MSCs are still associated with significant controversy and uncertainty as a conventional infection treatment method.

\section{Conclusion}

In an acute inflammation response, MSCs will first migrate to the site of infection and then regulate their differentiation and immunological effects via the stimulation of inflammatory factors. When an acute infection shifts to a chronic inflammation, the immune regulatory function and osteogenic differentiation ability of MSCs will be changed, which will eventually lead to the persistence of inflammation, bone destruction, and nonunion. The differentiation and immune regulation ability of MSCs under infection is bidirectionally regulated through multiple pathways. The transformation between acute inflammation and chronic inflammation has become a crucial point of tissue repair. The behavior of MSCs in acute and chronic inflammation states is worthy of further study, which may reveal the key involvement of MSCs in the whole process of infection.

\section{Abbreviations}

MSCs: mesenchymal stem cells; ECM: extracellular matrix; TNF- $\alpha$ : tumor necrosis factor- $\alpha$; TGF- $\beta$ : transforming growth factor- $\beta$; BMP: bone morphogenetic protein; Ser: serine; Thr: threonine; MSK 1: mitogen and stress-activated protein kinases 1; MSK 2: mitogen and stress-activated protein kinases 2; CBFA1: core binding factor factor A1; PI3K: phosphoinositide 3-kinase; $\mathrm{PKB}$ : protein kinase $\mathrm{B}$; mTOR: mammalian target of rapamycin; GSK3b: glycogen synthase kinase-3b; TCF4: T-cell factor 4; LEF1: lymphoid enhancing factor-1; NF-kB: nuclear factor kappa-B; TLRs: Toll-like receptors; PRRs: pattern recognition receptors; PAMPs: pathogenassociated molecular pattern; DAMPs: damageassociated molecular patterns; LTA: lipoteichoic acid; LPS: lipopolysaccharide; ATP: adenosine triphosphate; NSAIDs: non-steroidal antiinflammatory drugs; MAPK: mitogen-activated protein kinase; ERK: extracellular regulated protein kinases; JNK: c-Jun N-terminal kinase; AP-1: activator protein-1; SP-1: transcription factor-1; BMP: bone 
morphogenetic protein; SMAD: mothers against decapentaplegic homolog; R-SMAD: receptoractivated mothers against decapentaplegic homolog; Co-SMAD: common pathway mothers against decapentaplegic homolog; I-SMAD: inhibitory mothers against decapentaplegic homolog; RUNX2: runt-related transcription factor 2; OSX: recombinant osterix; Th: helper $\mathrm{T}$ cells; CTL: cytotoxic $\mathrm{T}$ lymphocyte; Tregs: regulatory $\mathrm{T}$ cells; IL-4: interleukin-4; IL-6: interleukin-6; IL-10: interleukin-10; P2X7: purinergic receptor $\mathrm{p} 2 \mathrm{x}$, ligand gated ion channel, 7; HO: heterotopic ossification; Fra-1: Fos related antigen-1; NK: natural killer; DCs: dendritic cells; IFN- $\gamma$ : interferon- $\gamma$; TNF- $\beta$ : tumor necrosis factor- $\beta$; PGE2: prostaglandin E2; IDO: indoleamine 2,3-dioxygenase; HGF: hepatocyte growth factor; CCL2: C-C motif ligand 2; VEGF: vascular endothelial growth factor; M-CSF: macrophage colony stimulating factor; STAT3: activating signal transducers and activators of transcription 3 .

\section{Acknowledgements}

Tiecheng Yu conceived the article, the first draft of the manuscript was written by Feng Gu. Ke Zhang critically revised the work. Feng $\mathrm{Gu}$, Ke Zhang, Jiangbi Li, Xiaoping Xie, Qiangqiang Wen, Zhenjiang Sui, Zilong Su, performed the literature search and figure work. All authors read and approved the final manuscript.

\section{Competing Interests}

The authors have declared that no competing interest exists.

\section{References}

1. Kavanagh N, Ryan EJ, Widaa A, et al. Staphylococcal Osteomyelitis: Disease Progression, Treatment Challenges, and Future Directions. Clin Microbiol Rev. 2018;31(2)

2. Wei X, Yang X, Han ZP, Qu FF, Shao L, Shi YF. Mesenchymal stem cells: a new trend for cell therapy. Acta Pharmacol Sin. 2013;34(6):747-754.

3. Beeravolu N, Khan I, McKee C, et al. Isolation and comparative analysis of potential stem/progenitor cells from different regions of human umbilical cord. Stem Cell Res. 2016;16(3):696-711.

4. Kita K, Gauglitz GG, Phan TT, Herndon DN, Jeschke MG. Isolation and characterization of mesenchymal stem cells from the sub-amniotic human umbilical cord lining membrane. Stem Cells Dev. 2010;19(4):491-502.

5. Alcorta-Sevillano N, Macías I, Rodríguez CI, Infante A. Crucial Role of Lamin A/C in the Migration and Differentiation of MSCs in Bone. Cells. 2020;9(6)

6. Muncie JM, Weaver VM. The Physical and Biochemical Properties of the Extracellular Matrix Regulate Cell Fate. Curr Top Dev Biol. 2018;130:1-37.

7. Engler AJ, Sen S, Sweeney HL, Discher DE. Matrix elasticity directs stem cell lineage specification. Cell. 2006;126(4):677-689.

8. Lee SD, Choe JW, Lee BJ, et al. Butein effects in colitis and interleukin-6/signal transducer and activator of transcription 3 expression. World J Gastroenterol. 2015;21(2):465-474

9. Afrazi A, Branca MF, Sodhi CP, et al. Toll-like receptor 4-mediated endoplasmic reticulum stress in intestinal crypts induces necrotizing enterocolitis. J Biol Chem. 2014;289(14):9584-9599.

10. Sun $D$, Junger WG, Yuan $C$, et al. Shockwaves induce osteogenic differentiation of human mesenchymal stem cells through ATP release and activation of P2X7 receptors. Stem Cells. 2013;31(6):1170-1180.

11. Lukač N, Katavić V, Novak S, et al. What do we know about bone morphogenetic proteins and osteochondroprogenitors in inflammatory conditions? Bone. 2020;137:115403.

12. Sampath TK, Reddi AH. Discovery of bone morphogenetic proteins - A historical perspective. Bone. 2020;140:115548.
13. Katagiri $\mathrm{T}, \mathrm{Tsukamoto} \mathrm{S}$. The unique activity of bone morphogenetic proteins in bone: a critical role of the Smad signaling pathway. Biol Chem. 2013;394(6):703-714.

14. Schupbach D, Comeau-Gauthier M, Harvey E, Merle G. Wnt modulation in bone healing. Bone. 2020;138:115491.

15. Chen D, Xie R, Shu B, et al. Wnt signaling in bone, kidney, intestine, and adipose tissue and interorgan interaction in aging. Ann N Y Acad Sci. 2019;1442(1):48-60.

16. Da $\mathrm{Y}$, Mou $\mathrm{Y}$, Wang $\mathrm{M}$, et al. Mechanical stress promotes biological functions of $\mathrm{C} 2 \mathrm{C} 12$ myoblasts by activating PI3K/AKT/mTOR signaling pathway. Mol Med Rep. 2020;21(1):470-477.

17. Elbadawy M, Usui T, Yamawaki H, Sasaki K. Novel Functions of Death-Associated Protein Kinases through Mitogen-Activated Protein Kinase-Related Signals. Int J Mol Sci. 2018;19(10)

18. Han J, Wu J, Silke J. An overview of mammalian p38 mitogen-activated protein kinases, central regulators of cell stress and receptor signaling. F1000Res. 2020;9.

19. Wagner EF. Functions of AP1 (Fos/Jun) in bone development. Ann Rheum Dis. 2002;61 Suppl 2(Suppl 2):ii40-42.

20. Katagiri T, Watabe T. Bone Morphogenetic Proteins. Cold Spring Harb Perspect Biol. 2016;8(6)

21. Hill CS. Nucleocytoplasmic shuttling of Smad proteins. Cell Res. 2009;19(1):36-46.

22. Duan Y, Haybaeck J, Yang Z. Therapeutic Potential of PI3K/AKT/mTOR Pathway in Gastrointestinal Stromal Tumors: Rationale and Progress. Cancers (Basel). 2020;12(10).

23. Schunk SJ, Floege J, Fliser D, Speer T. WNT- $\beta$-catenin signalling - a versatile player in kidney injury and repair. Nat Rev Nephrol. 2021;17(3):172-184.

24. Hayashi M, Nakashima T. [The bone and Wnt signaling : overview.]. Clin Calcium. 2019;29(3):277-282.

25. Cho TJ, Gerstenfeld LC, Einhorn TA. Differential temporal expression of members of the transforming growth factor beta superfamily during murine fracture healing. J Bone Miner Res. 2002;17(3):513-520.

26. Dimitriou R, Tsiridis E, Giannoudis PV. Current concepts of molecular aspects of bone healing. Injury. 2005;36(12):1392-1404.

27. Maruyama M, Rhee C, Utsunomiya T, et al. Modulation of the Inflammatory Response and Bone Healing. Front Endocrinol (Lausanne). 2020;11:386.

28. Kusadasi N, Groeneveld AB. A perspective on mesenchymal stromal cell transplantation in the treatment of sepsis. Shock. 2013;40(5):352-357.

29. Ponte AL, Marais E, Gallay N, et al. The in vitro migration capacity of human bone marrow mesenchymal stem cells: comparison of chemokine and growth factor chemotactic activities. Stem Cells. 2007;25(7):1737-1745.

30. Li Y, Liu YH, Li ZJ, et al. Staphylococcus aureus infection of intestinal epithelial cells induces human umbilical cord-derived mesenchymal stem cell migration. Int Immunopharmacol. 2013;15(1):176-181.

31. Durand N, Russell A, Zubair AC. Effect of Comedications and Endotoxins on Mesenchymal Stem Cell Secretomes, Migratory and Immunomodulatory Capacity. J Clin Med. 2019;8(4).

32. Ward LSC, Sheriff L, Marshall JL, et al. Podoplanin regulates the migration of mesenchymal stromal cells and their interaction with platelets. J Cell Sci. 2019;132(5).

33. Raicevic G, Najar M, Stamatopoulos B, et al. The source of human mesenchymal stromal cells influences their TLR profile as well as their functional properties. Cell Immunol. 2011;270(2):207-216.

34. Ward CL, Sanchez CJ, Jr, Pollot BE, et al. Soluble factors from biofilms of wound pathogens modulate human bone marrow-derived stromal cell differentiation, migration, angiogenesis, and cytokine secretion. BMC Microbiol. 2015;15:75.

35. Mastri M, Shah Z, McLaughlin T, et al. Activation of Toll-like receptor 3 amplifies mesenchymal stem cell trophic factors and enhances therapeutic potency. Am J Physiol Cell Physiol. 2012;303(10):C1021-1033.

36. Copuroglu C, Calori GM, Giannoudis PV. Fracture non-union: who is at risk? Injury. 2013;44(11):1379-1382

37. Foulke BA, Kendal AR, Murray DW, Pandit H. Fracture healing in the elderly: A review. Maturitas. 2016;92:49-55.

38. Andrzejowski P, Giannoudis PV. The 'diamond concept' for long bone non-union management. J Orthop Traumatol. 2019;20(1):21.

39. Liu X, Wang Y, Cao Z, et al. Staphylococcal lipoteichoic acid promotes osteogenic differentiation of mouse mesenchymal stem cells by increasing autophagic activity. Biochem Biophys Res Commun. 2017;485(2):421-426.

40. Wu T, Zhang J, Wang B, Sun Y, Liu Y, Li G. Staphylococcal enterotoxin C2 promotes osteogenesis of mesenchymal stem cells and accelerates fracture healing. Bone Joint Res. 2018;7(2):179-186.

41. Chang J, Liu F, Lee M, et al. NF-kB inhibits osteogenic differentiation of mesenchymal stem cells by promoting $\beta$-catenin degradation. Proc Natl Acad Sci U S A. 2013;110(23):9469-9474.

42. Lin TH, Gibon E, Loi F, et al. Decreased osteogenesis in mesenchymal stem cells derived from the aged mouse is associated with enhanced NF-KB activity. J Orthop Res. 2017;35(2):281-288.

43. Chen $\mathrm{X}, \mathrm{Hu} \mathrm{C}$, Wang $\mathrm{G}$, et al. Nuclear factor- $\mathrm{kB}$ modulates osteogenesis of periodontal ligament stem cells through competition with $\beta$-catenin signaling in inflammatory microenvironments. Cell Death Dis. 2013;4(2):e510.

44. Goodman SB, Pajarinen J, Yao Z, Lin T. Inflammation and Bone Repair: From Particle Disease to Tissue Regeneration. Front Bioeng Biotechnol. 2019;7:230. 
45. $\mathrm{Xu} X Y, \mathrm{He} X \mathrm{~T}$, Wang $\mathrm{J}$, et al. Role of the P2X7 receptor in inflammation-mediated changes in the osteogenesis of periodontal ligament stem cells. Cell Death Dis. 2019;10(1):20.

46. Li W, Li G, Zhang Y, et al. Role of P2 $\times 7$ receptor in the differentiation of bone marrow stromal cells into osteoblasts and adipocytes. Exp Cell Res. 2015;339(2):367-379.

47. Martin EC, Qureshi AT, Llamas CB, et al. Trauma induced heterotopic ossification patient serum alters mitogen activated protein kinase signaling in adipose stem cells. J Cell Physiol. 2018;233(9):7035-7044.

48. Miras-Portugal MT, Queipo MJ, Gil-Redondo JC, et al. P2 receptor interaction and signalling cascades in neuroprotection. Brain Res Bull. 2019;151:74-83.

49. Queipo MJ, Gil-Redondo JC, Morente V, et al. P2X7 Nucleotide and EGF Receptors Exert Dual Modulation of the Dual-Specificity Phosphatase 6 (MKP-3) in Granule Neurons and Astrocytes, Contributing to Negative Feedback on ERK Signaling. Front Mol Neurosci. 2017;10:448.

50. Wang YW, Xu DP, Liu Y, Zhang R, Lu L. The Effect of Tumor Necrosis Factor- $\alpha$ at Different Concentrations on Osteogenetic Differentiation of Bone Marrow Mesenchymal Stem Cells. J Craniofac Surg. 2015;26(7):2081-2085

51. Chen E, Liu G, Zhou X, et al. Concentration-dependent, dual roles of IL-10 in the osteogenesis of human BMSCs via P38/MAPK and NF-KB signaling pathways. Faseb j. 2018;32(9):4917-4929.

52. Agarwal S, Sorkin M, Levi B. Heterotopic Ossification and Hypertrophic Scars. Clin Plast Surg. 2017;44(4):749-755.

53. Sorkin M, Huber AK, Hwang C, et al. Regulation of heterotopic ossification by monocytes in a mouse model of aberrant wound healing. Nat Commun. 2020;11(1):722.

54. Evans KN, Potter BK, Brown TS, Davis TA, Elster EA, Forsberg JA. Osteogenic gene expression correlates with development of heterotopic ossification in war wounds. Clin Orthop Relat Res. 2014;472(2):396-404.

55. van der Kraan PM, Blaney Davidson EN, Blom A, van den Berg WB. TGF-beta signaling in chondrocyte terminal differentiation and osteoarthritis: modulation and integration of signaling pathways through receptor-Smads. Osteoarthritis Cartilage. 2009;17(12):1539-1545

56. Wang W, Rigueur D, Lyons KM. TGF $\beta$ signaling in cartilage development and maintenance. Birth Defects Res C Embryo Today. 2014;102(1):37-51.

57. Convente MR, Chakkalakal SA, Yang E, et al. Depletion of Mast Cells and Macrophages Impairs Heterotopic Ossification in an Acvr1(R206H) Mouse Model of Fibrodysplasia Ossificans Progressiva. J Bone Miner Res. 2018;33(2):269-282.

58. Kan L, Liu Y, McGuire TL, et al. Dysregulation of local stem/progenitor cells as a common cellular mechanism for heterotopic ossification. Stem Cells. 2009;27(1):150-156.

59. Torossian F, Guerton B, Anginot A, et al. Macrophage-derived oncostatin M contributes to human and mouse neurogenic heterotopic ossifications. JCI Insight. 2017;2(21).

60. Zhang Q, Zhou D, Wang H, Tan J. Heterotopic ossification of tendon and ligament. J Cell Mol Med. 2020;24(10):5428-5437.

61. Kim N, Cho SG. Overcoming immunoregulatory plasticity of mesenchymal stem cells for accelerated clinical applications. Int $\mathrm{J}$ Hematol. 2016;103(2):129-137.

62. Safar HA, Mustafa AS, Amoudy HA, El-Hashim A. The effect of adjuvants and delivery systems on Th1, Th2, Th17 and Treg cytokine responses in mice immunized with Mycobacterium tuberculosis-specific proteins. PLoS One. 2020;15(2):e0228381.

63. Joel MDM, Yuan J, Wang J, et al. MSC: immunoregulatory effects, roles on neutrophils and evolving clinical potentials. Am J Transl Res. 2019;11(6):3890-3904

64. Ma S, Xie N, Li W, Yuan B, Shi Y, Wang Y. Immunobiology of mesenchymal stem cells. Cell Death Differ. 2014;21(2):216-225.

65. Di Trapani M, Bassi G, Midolo $M$, et al. Differential and transferable modulatory effects of mesenchymal stromal cell-derived extracellular vesicles on T, B and NK cell functions. Sci Rep. 2016;6:24120.

66. Luz-Crawford P, Djouad F, Toupet K, et al. Mesenchymal Stem Cell-Derived Interleukin 1 Receptor Antagonist Promotes Macrophage Polarization and Inhibits B Cell Differentiation. Stem Cells. 2016;34(2):483-492.

67. Del Fattore A, Luciano R, Pascucci L, et al. Immunoregulatory Effects of Mesenchymal Stem Cell-Derived Extracellular Vesicles on T Lymphocytes. Cell Transplant. 2015;24(12):2615-2627.

68. Cho DI, Kim MR, Jeong HY, et al. Mesenchymal stem cells reciprocally regulate the M1/M2 balance in mouse bone marrow-derived macrophages. Exp Mol Med. 2014;46(1):e70

69. Wang $Y$, Han $B$, Wang $Y$, et al. Mesenchymal stem cell-secreted extracellular vesicles carrying TGF- $\beta 1$ up-regulate miR-132 and promote mouse M2 macrophage polarization. J Cell Mol Med. 2020;24(21):12750-12764.

70. Deng P, Zhou C, Alvarez R, Hong C, Wang CY. Inhibition of IKK/NF-кB Signaling Enhances Differentiation of Mesenchymal Stromal Cells from Human Embryonic Stem Cells. Stem Cell Reports. 2019;12(1):180-181.

71. Shi $Y$, Wang Y, Li Q, et al. Immunoregulatory mechanisms of mesenchymal stem and stromal cells in inflammatory diseases. Nat Rev Nephrol. 2018;14(8):493-507.

72. Nauta AJ, Kruisselbrink AB, Lurvink E, Willemze R, Fibbe WE. Mesenchymal stem cells inhibit generation and function of both CD34+-derived and monocyte-derived dendritic cells. J Immunol. 2006;177(4):2080-2087.

73. Spaggiari GM, Capobianco A, Becchetti S, Mingari MC, Moretta L. Mesenchymal stem cell-natural killer cell interactions: evidence that activated
NK cells are capable of killing MSCs, whereas MSCs can inhibit IL-2-induced NK-cell proliferation. Blood. 2006;107(4):1484-1490.

74. Petri RM, Hackel A, Hahnel K, et al. Activated Tissue-Resident Mesenchymal Stromal Cells Regulate Natural Killer Cell Immune and Tissue-Regenerative Function. Stem Cell Reports. 2017;9(3):985-998.

75. Munir H, Ward LSC, Sheriff L, et al. Adipogenic Differentiation of Mesenchymal Stem Cells Alters Their Immunomodulatory Properties in a Tissue-Specific Manner. Stem Cells. 2017;35(6):1636-1646.

76. Pajarinen J, Lin T, Gibon E, et al. Mesenchymal stem cell-macrophage crosstalk and bone healing. Biomaterials. 2019;196:80-89.

77. Saeedi P, Halabian R, Fooladi AAI. Antimicrobial effects of mesenchymal stem cells primed by modified LPS on bacterial clearance in sepsis. J Cell Physiol. 2019;234(4):4970-4986.

78. Yuan $\mathrm{Y}$, Lin $\mathrm{S}$, Guo N, et al. Marrow mesenchymal stromal cells reduce methicillin-resistant Staphylococcus aureus infection in rat models. Cytotherapy. 2014;16(1):56-63.

79. Sutton MT, Fletcher D, Ghosh SK, et al. Antimicrobial Properties of Mesenchymal Stem Cells: Therapeutic Potential for Cystic Fibrosis Infection, and Treatment. Stem Cells Int. 2016;2016:5303048.

80. Seebach E, Holschbach J, Buchta N, Bitsch RG, Kleinschmidt K, Richter W. Mesenchymal stromal cell implantation for stimulation of long bone healing aggravates Staphylococcus aureus induced osteomyelitis. Acta Biomater. 2015;21:165-177. 